agreement with our assessment. Genetic testing was subsequently positive for congenital myotonic dystrophy. Parents finally consented to withdrawal of intensive care at day 64 of life and he died shortly after extubation.

Conclusion Critical care decision on withdrawal of intensive care can be a very traumatic experience for families. It is essential to follow the guidance available. As paediatricians we are advocates for the baby but at the same time we have to be empathetic and considerate to the sentiments of the family.

\section{NECROTIZING ENTEROCOLITIS IN A NEWBORN FOLLOWING INTRAVENOUS IMMUNGLOBULIN TREATMENT FOR HEMOLYTIC DISEASE}

doi:10.1136/archdischild-2012-302724.0535

'S Kara, ${ }^{2} \mathrm{H}$ Ulu-Özkan, ${ }^{2 Y}$ Y YImaz, ${ }^{3} \mathrm{Fi}$ Arıkan, ${ }^{2} \mathrm{U}$ Dilmen, ${ }^{3} \mathrm{Y}$ Dallar Bilge. ${ }^{1}$ Ankara Training and Research Hospital, Department of Neonatology; 'Zekai Tahir Burak Maternity Training Hospital, Department of Neonatology: ${ }^{3}$ Ankara Training and Research Hospital, Department of Pediatrics, Ankara, Turkey

$\mathrm{ABO}$ iso-immunization is the most frequent hemolytic disease of the newborn. Treatment depends on the total serum bilirubin level, which may increase very rapidly in the first $48 \mathrm{~h}$ of life in cases of hemolytic disease of the newborn. Phototherapy and, in severe cases, exchange transfusion are used to prevent hyperbilirubinemia encephalopathy. Intravenous immunoglobulins are used to reduce exchange transfusion. Herein we present a female infant who was admitted to the our NICU because of ABO immune hemolytic disease and after two courses of $1 \mathrm{gr} / \mathrm{kg}$ of IVIG infusion, she developed NEC. Administration of IVIG to newborns with significant hyperbilirubinemia due to $\mathrm{ABO}$ hemolytic disease should be cautiously employed and always administered under strict.

\section{NEONATAL HSV ENCEPHALITIS: CONTROVERSIES OVER DIFFERENT THERAPEUTIC APPROACHES AND THEIR EFFECTS ON NEURO-DEVELOPMENTAL OUTCOMES}

doi:10.1136/archdischild-2012-302724.0536

A Papandreou, E Lek, A Pahuja, C Ramesh. Paediatrics, West Hertfordshire Hospitals NHS Trust, Watford, UK

Background and Aims Neonatal HSV encephalitis is well described and known to cause morbidity. However, there is no consensus regarding its optimal treatment, especially around using suppressive oral therapy after intravenous acyclovir. We aim to discuss treatment controversies and review possible neurodevelopmental outcomes in such cases.

Methods We report a case of vertically transmitted neonatal HSV-1 encephalitis and review existing literature on available treatment options (PubMed, EMBASE).

Results Our patient became pyrexial (39.0C) and lethargic on day 7 of life. Investigations revealed a raised CRP $(80 \mathrm{mg} / \mathrm{l})$ and CSF pleocytosis (WCC-26/ $\mathrm{mm}^{3}$, 90\% lymphocytes) with normal CSF biochemistry. IV antibiotics were empirically started. After developing encephalopathy and seizures on day 2 of illness, IV acyclovir was added. CSF PCR was positive for HSV-1. EEG showed multifocal irritability/excitability and asymmetrical temporal lobe activities. MRI showed low signal intensity on the ADC map in the medial temporal lobe cortices bilaterally and the right inferior frontal cortex.

21 days of IV acyclovir were completed, following which a repeat CSF sample was negative for HSV-1 PCR. IV antivirals were substituted with oral acyclovir at $1500 \mathrm{mg} / \mathrm{m}^{2} /$ dose BD for twelve months. Conclusions Literature review reveals controversies in treatment. Repeating HSV PCR at the end of IV treatment is not universally supported. Regarding suppressive oral acyclovir, some studies support doses of $1000-1740 \mathrm{mg} / \mathrm{m}^{2} /$ dose BD while others favour a
$300 \mathrm{mg} / \mathrm{m}^{2} /$ dose TDS regime. Its optimal duration (6months, 12 months or longer) is unclear. Neurodevelopmental outcomes mostly depend on the severity of the initial insult; Evidence that different suppressive treatments influence outcomes is poor.

\section{AN IMPORTANT CAUSE OF DYSKINESIA}

doi:10.1136/archdischild-2012-302724.0537

C Peevers. Paediatric Neurology, Bristol Children's Hospital, Bristol, UK

Kernicterus has been referred to a disease of the past. However, we report two cases of kernicterus presenting with a dyskinetic movement disorder. Both cases had neonatal jaundice and were well until the age of 3 years with normal intellect. On examination dystonia, dyskinesia and chorea were seen. Further examination revealed an upgaze palsy and auditory neuropathy.

Kernicterus describes a neurological syndrome resulting from deposition of unconjuated bilirubin in basal ganglia \& brainstem nuclei.

With the recent NICE guidance for jaundice therapy these cases highlight the importance of rigorous treatment of hyperbilirubinaemia. They also remind us to consider kernicterus as a diagnosis in a child presenting with a movement disorder and normal intellect.

\section{THE VALUE OF NEAR-INFRARED SPECTROSCOPY (NIRS) IN PERINATAL ASPHYXIA-A CASE REPORT}

doi:10.1136/archdischild-2012-302724.0538

${ }^{1} \mathrm{~F}$ Norooz, ${ }^{2 B}$ Urlesberger, ${ }^{1} \mathrm{~K}$ Klebermasz-Schrehof, ' $\mathrm{C}$ Czaba, ${ }^{1} \mathrm{E}$ Obwegeser, 'V Giordano, 'A Berger, 'M Weninger, 'M Olischar. 'Division of Paediatric Neonatology, Intensive Care and Neuropaediatrics, Medical University of Vienna, Vienna; ${ }^{2}$ Department of Paediatrics and Adolescent Medicine, Division of Neonatology, Medical University of Graz, Graz, Austria

Background Perinatal asphyxia remains a challenging entity. NIRS offers a method to continuously monitor cerebral oxygen saturation.

Aim To obtain insight into haemodynamic changes during hypothermia and rewarming in perinatal asphyxia using NIRS.

Methods We report of an asphyxiated patient $(37+6$ weeks', Apgar 6 and 8 at 1 and 5 minutes, first arterial blood gas $\mathrm{pH}$ of 6.67 , base deficit -25). NIRS was started during the first hour of life and continued for a total recording time of 125 hours. Simultaneously, we measured brain function using amplitude-integrated electroencephalography (aEEG). On day 7 magnetic resonance imaging (MRI) has been performed. After discharge, the patient was reassessed neurologically.

Results The initial cerebral $\mathrm{rSO} 2$ was $65 \%$. When cooling was started FTOE was 0.28 . At $33.5^{\circ} \mathrm{C}$ FTOE had decreased to 0.20 , cerebral $\mathrm{rSO} 2$ increased to $70 \%$. After rewarming cerebral $\mathrm{rSO} 2$ was $85 \%$, and FTOE 0.11. Initially, aEEG showed a mixed burst-suppression and discontinuous pattern which improved to a discontinuous pattern only during the first 12 hours. After rewarming aEEG normalized and showed developing sleep-wake cycles. MRI did not show any signs of hypoxic damage. After discharge the patient presented neurodevelopmentally normal.

Conclusion After having cooled down the patient, both NIRS and aEEG showed an improvement (increase of rSO2, decrease of FTOE, loss of burst-supression in aEEG). aEEG displays cerebral function, cerebral NIRS expands information to cerebral oxygen supply and extraction. MRI and neurodevelopmental assessment proved the observed aEEG and NIRS data.

539 SEVERE FORM OF CONGENITAL TOXOPLASMOSIS WITH EXTENSIVE CEREBRAL FINDINGS

doi:10.1136/archdischild-2012-302724.0539 